\title{
Síndrome de Mirizzi que simulaba una neoplasia biliar maligna
}

\author{
Mirizzi syndrome simulating a biliary malignant neoplasm
}

\author{
Freddy Pereira-Graterol D, Francisco Salazar-Marcano (D), Yajaira Venales-Barrios (D) \\ Unidad de Cirugía de Mínimo Acceso y Servicio de Cirugía General, Hospital Universitario "Dr. Luis Razetti", Barcelona, Venezuela
}

\begin{abstract}
Resumen
El síndrome de Mirizzi puede cursar con manifestaciones clínicas y hallazgos imagenológicos y de laboratorio, similares a los encontrados en las neoplasias biliares malignas.

Se describe el caso de un paciente cuyo enfoque clínico inicial y estudios de imágenes aportaron datos sugerentes de neoplasia de las vías biliares. Los marcadores tumorales (CA I9-9, CEA) resultaron elevados. Una nueva evaluación clínica, la laparoscopia exploradora y la exploración endoscópica de las vías biliares, permitieron establecer el diagnóstico de síndrome de Mirizzi. Después de la extracción del cálculo biliar y de la anastomosis entre el conducto hepático y el yeyuno, el paciente permaneció asintomático. En este caso, la reevaluación clínica y el uso del protocolo adecuado de estudio, contribuyeron a orientar y confirmar el diagnóstico de síndrome de Mirizzi, lo cual determinó la conducta terapéutica más acertada.

Palabras clave: Síndrome de Mirizzi; conducto colédoco; ictericia obstructiva; neoplasias; colangiopancreatografía retrógrada endoscópica; procedimientos quirúrgicos mínimamente invasivos.
\end{abstract}

\begin{abstract}
Mirizzi syndrome can occur with clinical manifestations and imaging and laboratory findings, similar to those found in malignant biliary neoplasms. We describe the case of a patient whose initial clinical approach and imaging studies provided data suggestive of bile duct neoplasia. Tumor markers (CA 19-9, CEA) were high. A new clinical evaluation, an explorative laparoscopy and endoscopic bile duct exploration allowed establish the diagnosis of Mirizzi syndrome. After removal the bile duct stone and to perform an hepatico-jejunostomy the patient remained asymptomatic. In this case the clinical reevaluation and the use of the appropriate study protocol contributed to guide and confirm the diagnosis of Mirizzi syndrome, which determined the most successful therapeutic approach.
\end{abstract}

Key words: Mirizzi Syndrome; common bile duct; jaundice, obstructive; neoplasms; cholangiopancreatography, endoscopic retrograde; minimally invasive surgical procedures.

Fecha de recibido: 27/06/2019 - Fecha de aceptación: 29/08/2019

Correspondencia: Freddy Pereira, Day Hospital \# 5, Avenida Principal de Lechería, Estado Anzoátegui, CP 6016, Venezuela.

Teléfono: (58) (416) 613-8797. Correo electrónico: freddypereiragraterol@gmail.com

Citar como: Pereira-Graterol F, Salazar-Marcano F, Venales-Barrios Y. Síndrome de Mirizzi que simulaba una neoplasia biliar maligna. Rev Colomb Cir. 2020;35:507-13. https://doi.org/10.30944/20117582.730

Este es un artículo de acceso abierto bajo una Licencia Creative Commons - BY-NC-ND https://creativecommons.org/licenses/by-ncnd/4.0/deed.es 


\section{Introducción}

La presentación del síndrome de Mirizzi varía desde la compresión extrínseca de la vía biliar hasta una fístula, colecisto-biliar, colecisto-entérica o ambas, con presencia de íleo biliar. A nivel internacional, se describe una incidencia de 0,05 a $4 \%$ en los pacientes sometidos a colecistectomía ${ }^{1,2}$ y, en Latinoamérica, alcanza el 5,7 \% y afecta principalmente a las mujeres (relación de 7 a 3) con edades comprendidas entre los 53 y los 70 años 3 . En Colombia, Díaz, et al., reportaron una incidencia de $3,5 \%$ de síndrome de Mirizzi en una muestra de 300 pacientes a quienes se les había practicado colecistectomía por laparoscopia 4; una cifra similar fue descrita por Aldana, et al., en I.234 pacientes con el mismo procedimiento, y observaron un porcentaje mayor con la colecistectomía de urgencia en comparación con la programada $(4,3 \text { Vs. } 2 \%)^{5}$.

El síndrome de Mirizzi y algunas neoplasias malignas de la vesícula y de las vías biliares, sobre todo en los estadios iniciales, pueden presentar un comportamiento clínico y hallazgos de imágenes similares. Asimismo, los niveles séricos de antígeno carbohidratado I9-9 (CA 19-9), glucoproteína asociada a las neoplasias malignas hepato-bilio-pancreáticas, gástricas, ováricas y colorrectales, pueden elevarse en ambas entidades; la ictericia obstructiva es la causa más común de falsos positivos ${ }^{6}$.

Se describe con detalle la estrategia empleada para el diagnóstico y el tratamiento de un paciente con síndrome de Mirizzi de tipo IV, en quien se sospechó inicialmente una neoplasia biliar.

\section{Caso clínico}

Se trata de un hombre de 56 años de edad, con antecedentes familiares de neoplasias malignas (madre fallecida por cáncer de cuello uterino, padre fallecido por cáncer de pulmón). Negó antecedentes personales médicos o quirúrgicos relevantes, así como ingestión alcohólica o tabaquismo.

Consultó por un cuadro clínico de ictericia, coluria, hipocolia y pérdida progresiva de peso $(8 \mathrm{~kg})$, de dos meses de evolución, no asociado a fiebre, prurito, emesis, melena o dolor abdominal. Inicialmente, consultó a un centro médico de otra localidad, en donde plantearon "neoplasia maligna biliar" fundamentados en los hallazgos de laboratorio, la colangiopancreatografía retrógrada endoscópica y la colangiorresonancia (figura I), y fue remitido a nuestro servicio en el Hospital Universitario “Dr. Luis Razetti”.

Durante la primera semana de hospitalización, se observó un patrón oscilante en la intensidad del tinte ictérico y la coluria, apreciándose dolor moderado a la palpación profunda del hipocondrio derecho, sin aumento de volumen ni signos de irritación peritoneal.

En los exámenes de laboratorio de ingreso se encontró: hemoglobina, I2,3 g/dL; hematocrito, $33 \%$ y leucocitos, 9.500 por $\mathrm{mm}^{3}$ (neutrófilos, $60 \%$ ); bilirrubina total, 3,5 $\mathrm{mg} / \mathrm{dL}$ (directa,
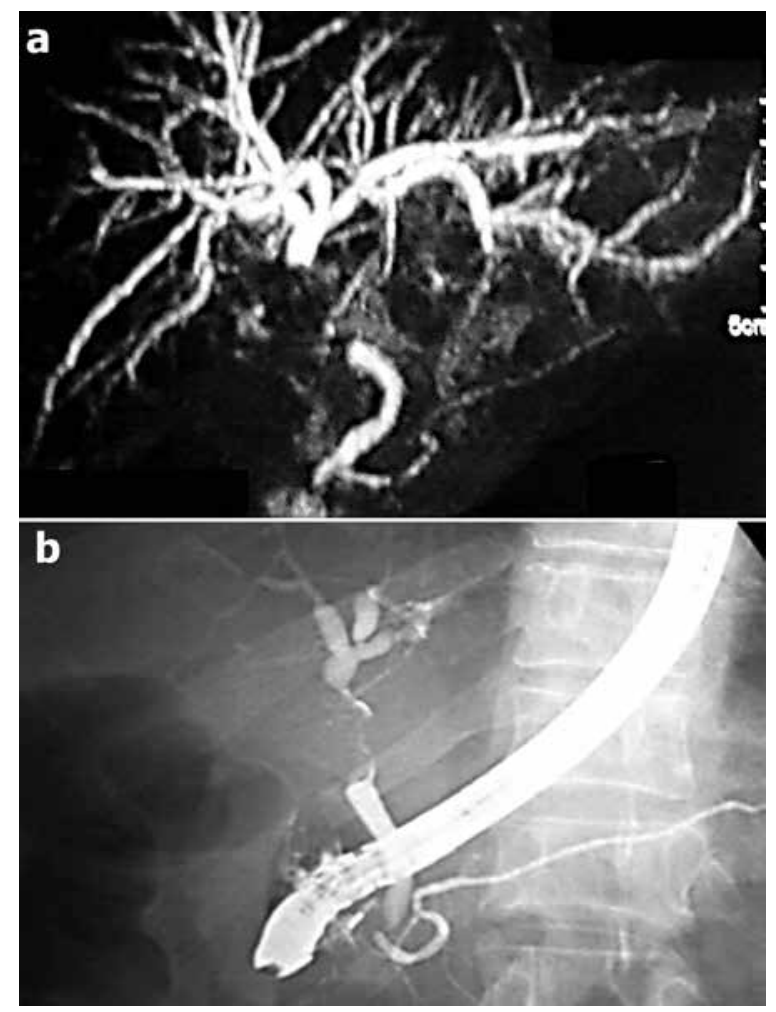

Figura 1. (a) Colangiorresonancia: dilatación de vías biliares intrahepáticas, con ausencia de señal en el tercio proximal de la vía biliar principal. (b) Colangiopancreatografía retrógrada endoscópica que demuestra defecto de llenado, irregular, en el mismo segmento de la vía biliar. 
I,6 g/dL); fosfatasa alcalina: $668 \mathrm{U} / \mathrm{L}$, ALT I39 U/L, AST I24 U/L; CA I9-9, 248 U/ml (normal: hasta 33 $\mathrm{U} / \mathrm{ml}$ ), y CEA, $7,8 \mathrm{ng} / \mathrm{ml}$ (normal: fumadores $\leq 2,5$ $\mathrm{ng} / \mathrm{ml}$, no fumadores $\leq 3,4 \mathrm{ng} / \mathrm{ml}$ ); además, la serología para hepatitis viral (A, B y C) fue negativa.

Después de una nueva evaluación clínica, se solicitó tomografía abdominal trifásica, nueva colangiografía endoscópica, citología biliar por cepillado, biopsia con pinza (figura 2) y laparoscopia exploradora durante el mismo acto anestésico; estos dos últimos procedimientos fueron realizados por el mismo equipo de especialistas del servicio.

En la colangiografía endoscópica se observó una imagen bien definida de ausencia de llenado en el tercio proximal de la vía biliar principal, no móvil, de aproximadamente $3 \mathrm{~cm}$ y con concavidad de superficie regular y dilatación de las vías biliares proximales a la obstrucción (figura 2), por lo cual se colocó una prótesis biliar plástica. La citología y la biopsia de la vía biliar fueron negativas para neoplasia maligna.
Durante la laparoscopia exploradora, se observó fusión entre la vesícula y la vía biliar principal e incremento de la consistencia de sus paredes, descartándose la presencia de lesiones de aspecto tumoral, metastásicas o adenomegalias, con lo cual se fundamentó el diagnóstico de síndrome de Mirizzi.

Mediante laparotomía, se extrajo el cálculo biliar y se hizo una anastomosis en Y de Roux entre el conducto hepático y el yeyuno; además, se apreció compromiso de más de dos tercios de las paredes de la vía biliar principal (figura 3).

La evolución posoperatoria fue satisfactoria; la vía oral se inició a las 48 horas y el egreso fue al quinto día. Igualmente, se observó mejoría de sus condiciones generales, normalización de las pruebas de laboratorio y ganancia de $\mathrm{I} 5 \mathrm{~kg}$ de peso corporal. A los 72 meses de seguimiento, el paciente permanecía asintomático, con ultrasonido abdominal y función hepática normales, y con persistencia de la permeabilidad del flujo bilio-entérico.
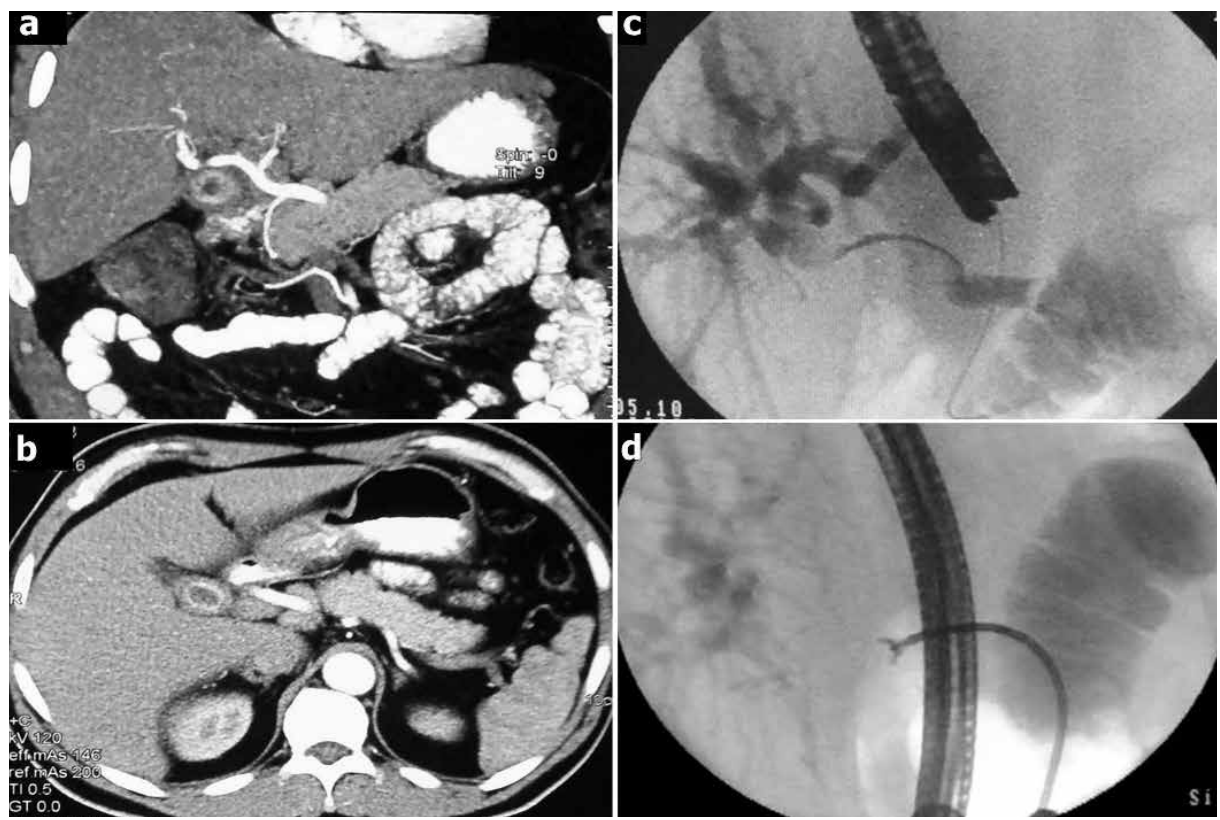

Figura 2. Tomografía computarizada trifásica de abdomen que demuestra un cálculo biliar, sin evidencia de lesiones tumorales o metastásicas en el hígado: corte coronal (a) y axial (b). Exploración endoscópica de las vías biliares: (c) colangiografía endoscópica. (d) toma de biopsia de la vía biliar para estudio de citología. 


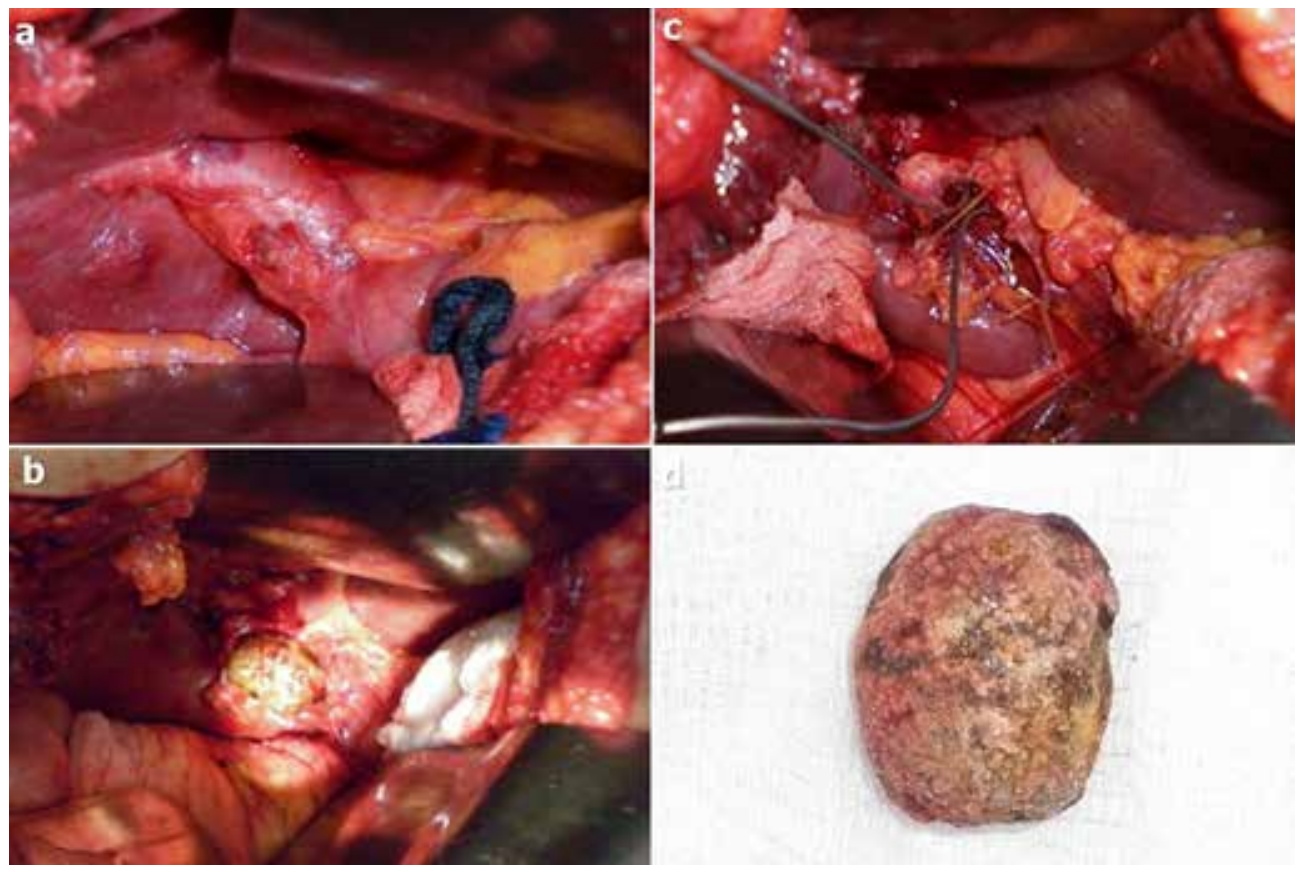

Figura 3. (a) Laparotomía: fusión entre la pared de la vesícula biliar y la vía biliar principal. (b) Fístula colecisto-biliar y cálculo de $3 \mathrm{~cm}$ en el interior de la vía biliar principal. (c) Compromiso de más de dos tercios del perímetro de la vía biliar principal. (d) Características macroscópicas del cálculo biliar que originó el síndrome de Mirizzi

\section{Discusión}

El síndrome de Mirizzi es una complicación inflamatoria crónica de la colecistolitiasis, en la cual los estudios de imágenes pueden demostrar alteraciones similares a las observadas en algunas neoplasias malignas biliares ${ }^{\mathrm{I}}$. Esta entidad, originada por la compresión sobre la vía biliar y compromiso de sus paredes, producidos por un cálculo localizado dentro de la vesícula, fue descrita inicialmente por Kehr (1905) y Ruge (1908) y, luego, detallada por Mirizzi (1948) ${ }^{3}$. Las consecuencias del proceso inflamatorio en el síndrome de Mirizzi van desde colestasis, por compresión extrínseca de la vía biliar, hasta fístulas colecisto-biliares (con diferentes grados de afectación de las paredes de la vía biliar) colecisto-entérica o ambas, con presencia en ocasiones de íleo biliar.

Con base en lo anterior, se han planteado diferentes clasificaciones. Inicialmente, Mirizzi describió el síndrome que hoy lleva su nombre como una compresión extrínseca de la vía biliar ${ }^{7}$.
Starling lo subdividió en tipo IA: conducto cístico largo que corre paralelo a la vía biliar, obstruido por cálculos, y tipo Ib: conducto cístico corto, completamente obliterado por cálculos, sin fístula ${ }^{8}$. McSherry (1982) ${ }^{9}$, Csendes (1989) ${ }^{\text {Io }}$, Beltrán y Csendes (2008) ${ }^{\text {II }} \mathrm{y}$, finalmente, Beltrán (20I2) ${ }^{3}$, plantearon clasificaciones más detalladas, siendo las de Csendes y Beltrán las más frecuentemente utilizadas; la propuesta por Payá-Llorente, et al., fue la más recientemente publicada ${ }^{12}$ (tabla I).

El caso descrito se agrupa dentro del tipo IV de la clasificación de Csendes ${ }^{\text {Io }}$, cuya incidencia es, aproximadamente, 3 de por cada Ioo pacientes con síndrome de Mirizzi, según lo reportado por Rodríguez, et al. ${ }^{13}$, y Beltrán, et al. ${ }^{3}$.

Por otra parte, la ictericia obstructiva puede ser una manifestación clínica común en enfermedades biliares benignas y malignas. En el síndrome de Mirizzi, la ictericia y el dolor en el hipocondrio derecho se describen en más de la mitad de los casos y, ocasionalmente, los 
Tabla 1. Clasificación del síndrome de Mirizzi según diversos autores. Se evalúa el compromiso de la superficie de la vía biliar, la fístula colecisto-biliar, la fístula colecisto-entérica y el íleo biliar

\begin{tabular}{|c|c|c|c|c|}
\hline & $\begin{array}{c}\text { Compresión } \\
\text { extrínseca de la vía biliar }\end{array}$ & $\begin{array}{c}\text { Fístula } \\
\text { colecisto-biliar }\end{array}$ & $\begin{array}{c}\text { Fístula } \\
\text { colecisto-entérica }\end{array}$ & Íleo biliar \\
\hline Mirizzi $(1948)^{7}$ & I & -- & -- & -- \\
\hline Starling $(1980)^{8}$ & $\begin{array}{l}\text { la: cístico largo, paralelo } \\
\text { a la vía biliar } \\
\text { lb: cístico corto obstruido }\end{array}$ & II & -- & -- \\
\hline \multirow[t]{2}{*}{ McSherry $(1982)^{9}$} & I & II & -- & -- \\
\hline & \multicolumn{3}{|c|}{ Compromiso de la vía biliar } & \\
\hline Csendes (1989) ${ }^{10}$ & I & $\begin{array}{l}\text { II: }<1 / 3 \\
\text { III: }<2 / 3 \\
\text { IV: }>2 / 3\end{array}$ & -- & -- \\
\hline Beltrán (2008) ${ }^{11}$ & I & $\begin{array}{l}\text { II: }<1 / 3 \\
\text { III: }<2 / 3 \\
\text { IV: }>2 / 3\end{array}$ & $\mathrm{Va}$ & $\mathrm{Vb}$ \\
\hline Beltrán $(2012)^{3}$ & I & $\begin{array}{l}\text { Ila: }<50 \% \\
\text { Ilb: }>50 \%\end{array}$ & IIla & IIIb \\
\hline Payá (2017) ${ }^{12}$ & $1 \mathrm{~A}$ & $\begin{array}{l}2 \mathrm{~A}:<50 \% \\
3 \mathrm{~A}:>50 \%\end{array}$ & $\begin{array}{l}1 \mathrm{~B} \\
2 \mathrm{~B} \\
3 \mathrm{~B}\end{array}$ & $\begin{array}{l}1 \mathrm{C} \\
2 \mathrm{C} \\
3 \mathrm{C}\end{array}$ \\
\hline
\end{tabular}

pacientes presentan inicialmente colangitis aguda ${ }^{14}$. Aunque por lo general el diagnóstico de síndrome de Mirizzi se hace intraoperatoriamente, su confirmación en el período preoperatorio permite planificar la estrategia quirúrgica más adecuada, para lo cual se requiere tanto de la sospecha clínica, como del apoyo en estudios de imágenes ${ }^{55,16}$.

La exploración endoscópica de las vías biliares y la colangiorresonancia se consideran el método diagnóstico estándar ${ }^{14}$. Con la combinación de la colangiorresonancia y la tomografía computarizada abdominal, se alcanza una sensibilidad del $96 \%$, una especificidad del $93,5 \%$, un valor predictivo positivo del $83,5 \%$ y una precisión del $94 \%{ }^{17}$. Un defecto excéntrico o la excavación en la pared lateral de la vía biliar principal a la altura del conducto cístico o la bolsa de Hartmann, la exclusión de la vesícula biliar y la dilatación de vía biliar proximal a la obstrucción son hallazgos frecuentes en los estudios de imágenes de los pacientes con síndrome de Mirizzi. Estas alteraciones también se pueden apreciar en el carcinoma de la vesícula biliar o el de las vías biliares ${ }^{1}$. El ultrasonido endoscópico tiene una sensibilidad del $95 \%$ y una especificidad del $97 \%$ para la detección de litiasis biliar ${ }^{\mathrm{I}}$; ; in embargo, hay escasos reportes sobre su uso en el diagnóstico del síndrome de Mirizzi ${ }^{19,20}$. Wehermann, et al., reportaron una sensibilidad diagnóstica del $97 \%$ y una especificidad del Ioo \% empleando el ultrasonido intraductal ${ }^{21}$.

En este paciente, aunque los estudios iniciales de imágenes (colangiorresonancia y colangiopancreatografía retrógrada endoscópica) orientaron hacia a una enfermedad biliar maligna, una nueva evaluación clínica enfocada en el patrón oscilante de la ictericia y la ausencia de sangrado reciente o antiguo en las heces, así como los posteriores estudios de extensión, permitieron establecer correctamente la etiología del cuadro clínico. La tomografía abdominal, la colangiografía endoscópica y, finalmente, la laparoscopia exploradora fueron herramientas fundamentales para el diagnóstico definitivo.

El CA 19-9 es una molécula de adhesión intracelular, sintetizada normalmente en el páncreas, la vía biliar, el estómago, el colon y el endometrio. 
La concentración sérica de esta glucoproteína puede aumentarse en las enfermedades biliares benignas (6I \%) o malignas $(86 \%)^{22}$, por lo que su medición no se debe considerar un método diagnóstico estándar para estas últimas, sino como un complemento útil durante su seguimiento. La sensibilidad de este marcador es del 80 al $90 \%$ en las neoplasias malignas del páncreas y del 60 al $70 \%$ en las neoplasias malignas de las vías biliares ${ }^{6}$.

El incremento de la presión en la vía biliar, que conlleva inflamación local, acumulación de CA I9-9 en la luz, aumento de la permeabilidad de la barrera entre bilis y sangre e incapacidad para degradar el antígeno en el hígado debido a la disfunción hepática, explican su elevación en presencia de obstrucción benigna de las vías biliares que condiciona colestasis, colangitis o ambas, al igual que durante los episodios de pancreatitis ${ }^{23}$.

La concentración sérica de este marcador tumoral puede ser influida por el estado secretor del paciente. Los pacientes con genotipo negativo para el antígeno Lewis (de grupo sanguíneo A o B negativo) que, en general, representan 4 a I5 \% de la población, no sintetizan el CA $19-9{ }^{24}$. En los pacientes con enfermedad biliar benigna, la concentración sérica de CA I9-9 se normaliza después de eliminar la causa de la obstrucción ${ }^{25}$. Incluso, se han reportado pacientes con síndrome de Mirizzi y elevación de este marcador tumoral por encima de $23.000 \mathrm{U} / \mathrm{ml}$, cuyos niveles se han normalizado después de resuelta la obstrucción biliar ${ }^{26-28}$. En el presente caso, con ictericia obstructiva, pérdida progresiva de peso y antecedentes familiares de neoplasias malignas, el CA I9-9 se encontró aumentado en siete veces su valor de referencia y se normalizó después del tratamiento quirúrgico.

El tratamiento del síndrome de Mirizzi depende del grado de compromiso de la vía biliar principal y de otros órganos afectados (en caso de fístula colecistoentérica, íleo biliar o ambos), y abarca una amplia variedad de técnicas quirúrgicas, desde la colecistectomía hasta la anastomosis bilio-entérica y resecciones gastro-entéricas ${ }^{29}$. En el presente caso, se exploraron las vías biliares y se practicó una anastomosis en Y de Roux hepático-yeyuno por tratarse de un síndrome de Mirizzi de tipo IV, con fusión de las paredes de la vesícula y la vía biliar principal. Esta conducta quirúrgica es similar a la recomendada por diversos autores, entre ellos, Rodríguez y Aldana del Hospital de San José (Bogotá), quienes la llevaron a cabo en dos pacientes con síndrome de Mirizzi, de tipo III en uno y de tipo IV en el otro ${ }^{13}$, y obtuvieron resultados satisfactorios.

Las conclusiones basadas en el reporte de un caso clínico poseen escaso nivel de evidencia, por lo que su aplicabilidad es baja; esto se convierte en una limitante en este artículo.

\section{Cumplimiento de normas éticas}

Consentimiento informado: Este estudio es una revisión de historia clínica retrospectiva y, como tal, no hay necesidad de un consentimiento informado.

Declaración de conflicto de intereses: ninguno declarado por los autores.

Fuentes de financiación: autofinanciado por los autores.

\section{Referencias}

I. Ibrarullah M, Mishra T, Das AP. Mirizzi syndrome. Indian J Surg. 2008;70:28I-7.

https://doi.org/I0.I007/sI2262-008-0084-y

2. Acquafresca P, Palermo M, Blanco L, García R, Tarsitano F. Síndrome de Mirizzi: prevalencia, diagnóstico y tratamiento. Acta Gastroenterol Latinoam. 20I4;44:323-8.

3. Beltrán MA. Mirizzi syndrome: History, current knowledge and proposal of a simplified classification. World J Gastroenterol. 2012;18:4639-50.

https://doi.org/I0.3748/wjg.vi8.i34.4639

4. Díaz S, Correa MJ, Giraldo LM, Ríos DC, Solórzano F, Wolff JD, et al. Experiencia en colecistectomía por laparoscopia en la Clínica Universitaria CES. Rev Colomb Cir. 2012;27:275-80.

5. Aldana GE, Martínez LE, Hosman MA, Ardila DA, Mariño IF, Sagra MR, et al. Factores predictores perioperatorios de complicaciones de la colecistectomía por laparoscopia. Rev Colomb Cir. 20I8;33:162-72. https://doi.org/IO.30944/201I7582.58

6. La Greca G, Sofia M, Lombardo R, Latteri S, Ricotta A, Puleo S, et al. Adjusting CAI9-9 values to predict 
malignancy in obstructive jaundice: Influence of bilirubin and C-reactive protein. World J Gastroenterol. 20I2;I8:4150-5.

https://doi.org/I0.3748/wjg.vi8.i3I.4I50

7. Mirizzi PL. Síndrome del conducto hepático. J Int Chir. I948;8:73I-77.

8. Starling JR, Matallana RH. Benign mechanical obstruction of the common hepatic duct (Mirizzi syndrome). Surgery. 1980;88:737-40.

9. McSherry CK, Ferstenberg H, Virshup M. The Mirizzi syndrome: Suggested classification and surgical therapy. Surg Gastroenterol. 1982;1:219-25.

Io. Csendes A, Díaz JC, Burdiles P, Maluenda F, Nava O. Mirizzi syndrome and cholecystobiliary fistula: A unifying classification. Br J Surg. 1989;76:1139-43. https://doi.org/IO.I002/bjs.I80076IIIO

II. Beltran MA, Csendes A, Cruces KS. The relationship of Mirizzi syndrome and cholecystoenteric fistula: Validation of a modified classification. World J Surg. 2008;32:2237-43. https://doi.org/I0.I007/s00268-008-9660-3

I2. Payá-Llorente C, Vázquez-Tarragón A, Alberola-Soler A, Martínez-Pérez A, Martínez-López E, Santarrufina-Martínez S. Mirizzi syndrome: A new insight provided by a novel classification. Ann Hepatobiliary Pancreat Surg. 20I7;21:67-75.

https://doi.org/IO.I470I/ahbps.20I7.21.2.67

I3. Rodríguez C, Aldana G. El síndrome de compresión biliar extrínseca benigna o síndrome de Mirizzi: experiencia de cinco años en el Hospital de San José. Rev Colomb Cir. 2008;23:6-II.

I4. Valderrama-Treviño AI, Granados-Romero JJ, Espejel-Deloiza M, Chernitzky-Camaño J, Barrera-Mera B, Estrada-Mata, AG, et al. Updates in Mirizzi syndrome. Hepatobiliary Surg Nutr. 20I7;6:170-8. https://doi.org/I0.21037/hbsn.20I6.II.OI

15. Kulkarni SS, Hotta M, Sher L, Maluenda F, Nava O. Complicated gallstone disease: Diagnosis and management of Mirizzi syndrome. Surg Endosc. 2017;31:2215-22. https://doi.org/I0.IO07/so0464-0I6-5219-9

I6. Chen H, Siwo EA, Khu M, Tian Y. Current trends in the management of Mirizzi syndrome: A review of literature. Medicine (Baltimore). 2018;97:e969I. https://doi.org/I0.I097/MD.oooooooooooo969I

17. Shanbhogue AK, Tirumani SH, Prasad SR, Fasih N, McInnes M. Benign biliary strictures: A current comprehensive clinical and imaging review. Am J Roentgenol. 20II;197:295-306. https://doi.org/ro.22I4/AJR.Io.6002

I8. Giljaca V, Gurusamy KS, Takwoingi Y, Higgie D, Poropat G, Štimac D, et al. Endoscopic ultrasound versus magnetic resonance cholangiopancreatography for common bile duct stones. Cochrane Database Syst
Rev. 20I5(2):CDoII549.

https://doi.org/IO.IOO2/I465I858.CDoII549

I9. Rayapudi K, Gholami P, Olyaee M. Mirizzi syndrome with endoscopic ultrasound image. Case Rep Gastroenterol. 2013;7:202-7. https://doi.org/I0.II59/00035II70

20. Peláez-Luna M, Levy MJ, Arora AS, Baron TH, Rajan E. Mirizzi syndrome presenting as painless jaundice: A rare entity diagnosed by EUS. Gastrointest Endosc. 2008;67:974-5.

https://doi.org/I0.IoI6/j.gie.2007.I2.00I

2I. Wehermann T, Riphaus A, Martchenko K, Kokabpick S, Pauka N, Stergiou N, et al. Intraductal ultrasonography in the diagnosis of Mirizzi syndrome. Endoscopy. 2006;38:717-22. https://doi.org/IO.IO55/s-2006-944524

22. Marrelli D, Caruso S, Pedrazzani C, Neri A, Fernandes E, Marini M. CAI9-9 serum levels in obstructive jaundice: Clinical value in benign and malignant conditions. Am J Surg. 2009;198:333-9. https://doi.org/IO.IoI6/j.amjsurg.2008.I2.03I

23. Ventrucci M, Pozzato P, Cipolla A, Uomo G. Persistent elevation of serum CA 19-9 with no evidence of malignant disease. Dig Liver Dis. 2009;4I:357-63. https://doi.org/IO.IoI6/j.dld.2008.04.002

24. Vestergaard EM, Hein HO, Meyer H, Grunnet N, Jørgensen J, Wolf $\mathrm{H}$, et al. Reference values and biological variation for tumor marker CA $19-9$ in serum for different Lewis and secretor genotypes and evaluation of secretor and Lewis genotyping in a Caucasian population. Clin Chem. 1999;45:54-6I.

25. Mann DV, Edwards R, Ho S, Lau WY, Glazer G. Elevated tumour marker CAI9-9: Clinical interpretation and influence of obstructive jaundice. Eur J Surg Oncol. 2000;26:474-9. https://doi.org/IO.IO53/ejso.I999.0925

26. Gibor U, Perry ZH, Netz U, Glazer Y, Laufer L, Kirshtein B. CA 19-9 in the presence of obstructive jaundice due to Mirizzi syndrome. Isr Med Assoc J. 2015;I7:60-I.

27. Shah N, Tetangco E, Arshad HMS, Raddawi H. Mirrizi syndrome and markedly elevated levels of carbohydrate antigen 19-9 in the absence of malignant disease. Case Rep Gastrointest Med. 2017;20I7:241690I https://doi.org/IO.II55/20I7/24I690I

28. Fontes PR, Teixeira UF, Waechter FL, Sampaio JA, Pereira-Lima L. Mirizzi syndrome in association with serum CA I9-9 greater than 20.000U/ml: Is it possible? Arq Bras Cir Dig. 2012;25:69-70. https://doi.org/IO.I590/SoI02-67202012000I000I7

29. Kumar A, Senthil G, Prakash A, Behari A, Kumar Singh R, Kumar Kapoor V, et al. Mirizzi's syndrome: Lessons learnt from I69 patients at a single Compresión center. Korean J Hepatobiliary Pancreat Surg. 2016;20:17-22. https://doi.org/IO.I470I/kjhbps.20I6.2O.I.I7 